\title{
Artigo/Article
}

\section{Perfil clínico-laboratorial de crianças vivendo com HIV/AIDS por transmissão vertical em uma cidade do Nordeste brasileiro}

\author{
Clinical and laboratory profile of children living with vertically transmitted HIV/AIDS in a \\ city in northeastern Brazily
}

\author{
Margareth Jamil Maluf e Silva ${ }^{1}$, Wellington Da Silva Mendes ${ }^{2}$, Mônica Elinor Alves Gama ${ }^{3}$, Maria Bethânia da \\ Costa Chein ${ }^{3}$ e Daniele Soares Veras ${ }^{4}$
}

\begin{abstract}
RESUMO
Introdução: A transmissão vertical constitui a principal via de infecção infantil pelo vírus HIV-1 (vírus da imunodeficiência humana). A presente pesquisa tem como objetivo estudar a evolução clínica e laboratorial de crianças vivendo com HIV/AIDS decorrente da transmissão vertical. Métodos: Trata-se de um estudo descritivo, retrospectivo, realizado a partir da coleta de dados em prontuário médico de todas as crianças atendidas em um Serviço de Assistência Especializada, no período de janeiro de 1998 a junho de 2006. Resultados: Foram avaliadas 80 crianças que preencheram critérios de inclusão. Observou-se que em 56 (70\%) crianças, o diagnóstico da infecção pelo HIV na mãe deu-se após o parto e que em 44 (55\%) o parto foi via vaginal. Amamentação ao seio materno foi documentada em 56 (70\%) crianças e esta variou de um mês até mais de 12 meses. A não utilização ou uso incompleto do Protocolo ACTG 076 foi documentado em 63 (78,5\%) casos. Conclusões: Os dados observados em nosso estudo são bastante preocupantes e revelam falha na assistência materno-infantil, especialmente voltada para prevenção da transmissão.
\end{abstract}

Palavras-chaves: HIV. AIDS. Transmissão vertical. Crianças.

\begin{abstract}
Introduction: Vertical transmission constitutes the main route for child infection by the HIV-1 virus (human immune deficiency virus). This study aimed to investigate the clinical and laboratory evolution of children with vertically transmitted HIV/AIDS. Methods: This was a retrospective descriptive study based on data gathered from the medical records of all the children who were seen at a specialized care unit between January 1998 and June 2006. Results: Eighty children who met the inclusion criteria were evaluated. In the cases 56 (70\%) of the children, their mothers were diagnosed as HIV-positive after childbirth. The delivery was vaginal for 44 (55\%) of the children. Fifty-six children (70\%) were breastfed by their mothers for periods ranging from one to more than 12 months. Failure to use or incomplete use of the ACTG 076 protocol was documented in 63 (78.5\%) of the cases. Conclusions: The findings from our study are a cause for considerable concern and show failures of medical care for mothers and children, particularly with regard to prevention of transmission.
\end{abstract}

Key-words: HIV. Aids. Vertical transmission. Children.

1. Departamento Materno Infantil, Hospital Universitário Materno Infantil, Universidade Federal do Maranhão. São Luis, MA. 2. Departamento de Patologia, Universidade Federal do Maranhão. São Luis, MA. 3. Departamento de Medicina III, Universidade Federal do Maranhão. São Luis, MA. 4. Curso de Medicina, Universidade Federal do Maranhão. São Luis, MA.

Endereço para correspondência: Dra. Margareth Jamil Maluf e Silva. Rua das Juçaras, Quadra 44, Casa 06, Renascença, 65075-230 São Luis, MA.

Tels: 5598 9975-4020/3235-6323

e-mail:margamjs@hotmail.com

Recebido para publicação em 20/04/2009

Aceito em 16/12/2009

\section{INTRODUÇÃO}

A síndrome da imunodeficiência adquirida (AIDS) foi identificada em 1981 e, desde então, tem se mantido endêmica no mundo. A AIDS tem afetado, em proporção cada vez maior, mulheres de todas as camadas sociais, especialmente aquelas que vivem em condição de pobreza e de baixa escolaridade $^{1}$. Ocorreu no Brasil um importante deslocamento no perfil da AIDS, incluindo cada vez mais mulheres de 15-40 anos em plena idade reprodutiva. Essa heterossexualização e feminização da epidemia têm numerosas conseqüências, dentre elas, o aumento do número de crianças infectadas pelo HIV tendo a transmissão vertical como principal via de infecção².

Diante dessa nova realidade, novas condutas foram incorporadas com vistas a redução da transmissão vertical do HIV. Em 1994, comprovouse a eficácia do Protocolo AIDS Clinical Trial Group 76 (ACTG 076) em um estudo multicêntrico realizado por Connor cols ${ }^{3}$, que demonstrou uma queda na transmissão vertical do HIV de $25,5 \%$ para $8,3 \%$, a partir da indicação do uso da zidovudina oral para gestante, endovenosa no parto e o xarope para o recém-nascido e a recomendação para alimentação artificial exclusiva; assim, traçouse um novo rumo para o controle da transmissão vertical do $\mathrm{HIV}^{4}$.

Porém, são reconhecidas dificuldades iniciais para incorporação progressiva dessas condutas nos diferentes serviços. Assim, no presente estudo, objetivou-se avaliar as características clínicas e laboratoriais das crianças infectadas pelo HIV por transmissão vertical no Serviço de Assistência Especializado (SAE) do Hospital Universitário da Universidade Federal do Maranhão - Unidade Materno-Infantil (HUMI), que se configura em uma referência no Estado do Maranhão para este atendimento. 


\section{MÉTODOS}

Foi desenvolvido um estudo descritivo, com componente retrospectivo, onde foram avaliadas as crianças vivendo com HIV/ AIDS, cuja infecção ocorreu por transmissão vertical, atendidas no SAE pediátrico do HUMI, no período de janeiro de 1998 a junho de 2006. A fonte principal de informação foi o prontuário médico das crianças cadastradas no serviço, a revisão foi realizada em Janeiro de 2007. Foram incluídas no estudo todas as crianças com diagnóstico de infecção pelo vírus HIV adquirida por transmissão vertical.

As variáveis avaliadas foram as características demográficas, o tipo de parto, o tempo de ruptura de bolsa, o aleitamento materno, o uso do protocolo ACTG 076, a classificação em categorias clínicas e o diagnóstico laboratorial.

As crianças foram classificadas de acordo com as categorias clínicas e imunológicas propostas pelo Centers for Diseases Control (CDC), Atlanta, EUA adotada pelo Ministério da Saúde ${ }^{4}$.

\section{Ética}

O estudo foi aprovado pelo Comitê de Ética em Pesquisa do Hospital Universitário da UFMA parecer número 198/05.

\section{RESULTADOS}

Oitenta crianças foram avaliadas no estudo, sendo 45 (56\%) do sexo masculino e 60 (75\%) com idade de 18 meses ou mais. Destas, 41 (51,3\%) tinham entre dois a seis anos no momento da avaliação. O diagnóstico de infecção por HIV na mãe ocorreu após o parto em 56 (70\%) casos. Em 16 (20\%) casos, esta informação não foi obtida (Tabela 1).

Segundo o tipo de parto, 44 (55\%) crianças nasceram de parto vaginal e em $23(28,7 \%)$ crianças esta informação não constava no prontuário. Duas crianças nasceram prematuras. Três crianças

TABELA 1 - Características demográficas e época do diagnóstico materno das crianças vivendo com HIV/AIDS. Hospital Universitário Materno Infantil, 1998-2006.

\begin{tabular}{lrr}
\hline Características & Número & Percentagem \\
\hline Idade (meses) & 20 & \\
$<18$ & 60 & 25,0 \\
$>18$ & & 75,0 \\
\hline Idade atual & 41 & 51,3 \\
pré-escolar & 20 & 25,0 \\
escolar & 14 & 17,5 \\
adolescente & 5 & 6,2 \\
\hline óbitos & & \\
\hline Sexo & 45 & 56,0 \\
masculino & 35 & 44,0 \\
feminino & & \\
\hline Diagnóstico materno de HIV & 4 & 5,0 \\
antes da gestação & 3 & 3,7 \\
durante gestação & 1 & 70,0 \\
durante o parto & 56 & 20,0 \\
após o parto & 16 & 100,0 \\
ignorados & 80 & \\
Total & & \\
\hline
\end{tabular}

HIV: human immunodeficiency virus. tiveram diagnóstico de sífilis congênita. Informações sobre a ruptura de membranas foram obtidas em apenas 28 (35\%) casos. Destas, 6 (21\%) ocorreram acima de 4 horas. Amamentação ao seio materno foi documentada em 56 (70\%) crianças e esta variou de um mês até mais de 12 meses. Em 19 (33,7\%) casos, a manutenção do aleitamento foi superior a 12 meses (Tabela 2).

TABELA 2 - Prevalência dos fatores de risco conhecidos para infecção pelo HIV. Hospital Universitário Materno Infantil, 1998-2006.

\begin{tabular}{|c|c|c|}
\hline Fatores de risco & Número & Percentagem \\
\hline \multicolumn{3}{|l|}{ Tipo de parto } \\
\hline cesárea & 13 & 16,3 \\
\hline vaginal & 44 & 55,0 \\
\hline ignorados & 23 & 28,0 \\
\hline total & 80 & 100,0 \\
\hline \multicolumn{3}{|c|}{ Protocolo ACTG 076} \\
\hline completo & 1 & 1,5 \\
\hline incompleto & 3 & 4 \\
\hline não usou & 63 & 78,5 \\
\hline ignorados & 13 & 16,0 \\
\hline total & 80 & 100,0 \\
\hline \multicolumn{3}{|c|}{ Tempo de ruptura das membranas (horas) } \\
\hline$<4$ & 23 & 28,8 \\
\hline$>4$ & 6 & 7,5 \\
\hline ignorados & 51 & 63,7 \\
\hline total & 80 & 100,0 \\
\hline \multicolumn{3}{|c|}{ Aleitamento materno } \\
\hline $\operatorname{sim}$ & 56 & 70,0 \\
\hline não & 5 & 6,3 \\
\hline ignorados & 19 & 23,7 \\
\hline total & 80 & 100,0 \\
\hline \multicolumn{3}{|c|}{ Tempo do aleitamento materno (meses) } \\
\hline$<1$ & 5 & 9,0 \\
\hline até 6 & 15 & 26,8 \\
\hline 7 a 12 & 17 & 30,3 \\
\hline$>12$ & 19 & 33,9 \\
\hline Total & 56 & 100,0 \\
\hline
\end{tabular}

Em relação ao Protocolo ACTG 076, foi documentada a não aplicação em $63(78,5 \%)$ casos, de forma completa ou incompleta. Em 13 (16\%) casos, esta informação não estava registrada no prontuário.

Considerando a classificação clínico-imunológica da HIV/AIDS, observou-se que das 80 crianças, 77 (96,2\%) eram sintomáticas e destas, 25 (33\%) foram classificadas na categoria B do CDC. Quanto à avaliação imune através da contagem de linfócitos TCD4+, $40(50 \%)$ crianças apresentavam imunodeficiência moderada e $22(27,5 \%)$ apresentavam imunodeficiência grave. Em 49 (61,2\%) casos, havia critérios para utilização de antirretrovirais.

\section{DISCUSSÃO}

Os dados observados em nosso estudo são bastante preocupantes e revelam uma grave falha na assistência materno-infantil, especialmente aquela voltada para prevenção da transmissão. Dentre elas, destacam-se a diagnóstico tardio de infecção pelo HIV em gestantes, a baixa aplicação do protocolo ACTG 076, a prática de 
aleitamento materno pelas gestantes infectadas pelo HIV e a não triagem para o tipo de parto.

Estes resultados observados suscitam que sejam levantados alguns questionamentos como: Houve atraso no início do pré-natal? Foram solicitados exames sorológicos para o HIV durante o pré-natal? Se foram realizados, os exames foram entregues em tempo hábil? O presente estudo não permite responder a esses questionamentos dado o limite das informações constantes nos prontuários, porém, deve-se destacar a importância do pré-natal para adoção das estratégias de redução na transmissão vertical do HIV.

O diagnóstico tardio da infecção pelo HIV na mãe, observado em nosso estudo, possivelmente, reflete um problema que possa estar ocorrendo em outras instituições. No Estudo Sentinela Parturiente, realizado em 2004, foi demonstrado que apenas 62,5\% das parturientes no Brasil tinham realizado o teste anti-HIV na gestação e conheciam o resultado do teste antes da internação para o parto. Na região Nordeste, foi observado que em $45,4 \%$ das gestantes não houve solicitação da sorologia anti-HIV 5 .

A elevada freqüência de parto vaginal, observada em nosso estudo, tem sido reportada por outros autores. No estudo de Yoshimoto $\mathrm{cols}^{6}$, foi documentado que 50\% dos partos foram por essa via. Tem sido observado que a maioria das transmissões verticais do HIV ocorre durante ou próximo ao período intraparto sugerindo que intervenções obstétricas, como parto cesariano, podem reduzir essas taxas. No início de 2005, o Estudo Colaborativo Europeu divulgou dados indicando que o parto cesariano pode reduzir a transmissão vertical do HIV-1, mesmo em pacientes com cargas virais inferiores a 1.000 cópias $/ \mathrm{ml}^{7}$.

O grande percentual de informação ignorada relativa ao tempo de ruptura de membranas, em nosso estudo, prejudica a interpretação desta variável. Tem sido demonstrado que quando ele ultrapassa 4 horas ocorre aumento significativo do risco de transmissão vertical ${ }^{8}$. Garcia cols ${ }^{9}$ mostraram uma taxa de transmissão de $7,5 \%$ e $23 \%$, respectivamente, conforme a ruptura ocorresse antes ou após 4 horas.

No SAE do HUMI, foi observada uma grande frequência de mães vivendo com HIV que amamentam seus filhos, algumas por até 12 meses. Diversos estudos têm demonstrado que o aleitamento materno pode ser associado com a transmissão vertical do HIV, podendo determinar um risco adicional de transmissão entre 7 e $22 \%^{10,11}$. Calcula-se que ocorram 8,9 transmissões por cada 100 crianças em um ano de aleitamento ${ }^{10}$. Um estudo na África não encontrou associação entre taxa de transmissão vertical do HIV e duração do aleitamento materno quando comparou amamentação por menos versus por mais de quatro semanas ${ }^{11}$.

Merece destaque o Projeto Nascer, criado em 2000, pelo Ministério da Saúde, com o foco na redução da transmissão do HIV; tem como objetivos, dentre outros, a testagem para o HIV e a garantia da fórmula infantil até os seis meses de idade ${ }^{9}$. No Estado do Maranhão, em 2000, o Projeto Nascer foi implantado, somente em uma maternidade; as demais, apenas em 2005, devendo-se considerar que o nascimento da população em estudo ocorreu entre 1993 e 2004, período em que não havia se dado à implantação efetiva das estratégias preconizadas pelo Projeto Nascer não estava implantado efetivamente ${ }^{13}$.

Desde 1994, estudos têm comprovado que o Protocolo ACTG 076 reduz a transmissão vertical do HIV de $25,5 \%$ para $8,3 \%{ }^{6,14}$. A despeito deste conhecimento, observamos que, no SAE do HUMI-
UFMA, em mais de $90 \%$ das gestantes ele não foi instituído de forma completa. Resultado diverso foi relatado por Vasconcelos cols ${ }^{15}$ que observaram em quatro cidades de diferentes regiões brasileiras que, $24 \%$ das gestantes e 7,6\% dos recém nascidos não haviam usado o Protocolo ACTG 076. Yoshimoto cols ${ }^{6}$ estudaram prospectivamente 64 recém-nascidos em São Paulo, e observam que no grupo de 20 crianças que usou o protocolo de forma incompleta três se infectaram, enquanto, no grupo que utilizou o protocolo completo, nenhum foi infectado.

A maioria das crianças, em nosso estudo, foi alocada na categoria B da classificação do CDC-EUA. Resultado diferente foi observado no estudo de Yoshimoto cols $^{6}$, onde $75 \%$ das crianças foram classificados na categoria $\mathrm{N}$.

A ocorrência de sífilis durante a gestação tem sido associadas ao aumento da transmissão do HIV ${ }^{6,9}$. No presente, estudo três crianças infectadas pelo HIV apresentavam concomitância de sífilis congênita.

A prematuridade, observada em dois de nossos pacientes, tem sido apontada como fator de risco para transmissão vertical do HIV-1. Duarte cols ${ }^{14}$ relatam que até $70 \%$ das mães com AIDS podem apresentar parto prematuro. Kuhn cols ${ }^{16}$ sugerem que o prematuro tem maior risco de adquirir infecção pelo HIV intraparto.

Considerando os dados do presente estudo, faz-se necessário destacar a relevância do cumprimento adequado das medidas definidas como eficazes para a garantia da redução da transmissão do HIV na população infantil, sob pena de se determinar grandes impactos sociais e econômicos em nosso país.

\section{AGRADECIMENTOS}

Ao Hospital Universitário Materno Infantil da Universidade Federal do Maranhão, e em particular à coordenação, docentes e colaboradores do Programa de Pós-Graduação em Saúde Materno Infantil Mestrado Acadêmico.

\section{CONFLITO DE INTERESSE}

Os autores declaram não haver nenhum tipo de conflito de interesse.

\section{SUPORTE FINANCEIRO}

Fundação de Amparo a Pesquisa do Estado do Maranhão (FAPEMA).

\section{REFERÊNCIAS}

1. Silva ANF. Estudo evolutivo das crianças expostas ao HIV e notificação pelo núcleo de vigilância epidemiológica do HCFMRP-USP. Dissertação de Mestrado, Universidade de São Paulo, Ribeirão Preto; 2004.

2. Joint United Nation Programme on Hiv/AIDS. AIDS epidemic update. Geneva, UNAIDS; 2006.

3. Connor EM, Sperling RS, Gelber R, Kiselev P, Scott G, O’Sullivan M, et al. Reducion of maternal-infant transmission of human immunodeficiency virus type 1 with zidovudine treatament. N Engl J Med 1994; 331: 1173-1180.

4. Ministério da Saúde. Guia de tratamento clinico da infecção pelo HIV em pediatria. Brasília, MS; 2006. 
5. Szwarwald CL. Estimativa do número de pessoas de 15 a 49 anos infectadas pelo HIV, Brasil, 2004. Boletim Epidemiológico AIDS 2006; 3: 11-15.

6. Yoshimoto CE, Diniz EMA, Vaz FAC. Evolução clínica e laboratorial de recémnascidos de mães HIV positivas. Rev Assoc Med Bras 2005; 51: 100-105.

7. European Collaborative Study. Mother-to-child transmission of HIV infection in the era of highly active antiretroviral therapy. Clin Infect Dis 2005; 40: 458-465.

8. Gianvecchio RP, Goldberg TBL. Fatores protetores e de risco envolvidos na transmissão vertical do HIV-1. Cad Saúde Pública 2005; 21: 581-588.

9. Garcia TA, Perales A, Maiques V. Duration of ruptured membranes and extended labor are risk factors for HIV transmission. Int J Gynecol Obstet 2003; 82: 17-23.

10. Rosseau MC, Nduati RW, Richardson AB, John-Stewart GC, Mbori-Ngacha AD, Kreiss JK et al. Association of levels of HIV-1-infected breast milk cells and risk of mother-to-child transmission. J Infect Dis 2004; 190: 1880-1888.

11. The Breastfeeding and H IVInternational Transmission Study Group. Late postnatal transmission of HIV-1 in breast-fed children: an individual patient data meta-analysis. J Infect Dis 2004; 189: 2154-2166.

12. Ministério da Saúde. Projeto Nascer. Série E: Comunicação em Saúde. Brasília, DF; 2003.

13. Secretaria de Saúde. Sistema de Informação de Agravos de Notificação. São Luís; 2006 .

14. Duarte G, Quintana SM, El Betune P. Estratégias que reduzem a transmissão vertical do vírus da imunodeficiência humana tipo 1. Rev Bras Ginecol Obstet 2005; 27: 768-778.

15. Vasconcelos ALR, Hamann EM. Por que o Brasil ainda registra elevados coeficientes de transmissão vertical do HIV? Uma avaliação da qualidade da assistência prestada a gestante/parturientes infectadas pelo HIV e seus recém nascidos. Rev Bras Saúde Mater Infant 2005; 4: 483-492.

16. Kuhn L, Abrams E, Matheson PB, Thomas PA, Lambert G, Bamji M et al. New York City Perinatal HIV Transmission Collaborative Study Group 9 AIDS. Cad Saúde Pública 1997; 11: 429-435. 